\title{
Ginsenoside Rc Protects Neurons From Oxygen- glucose Deprivation Injuries and Its Mechanistic Investigation via a Network Pharmacology-based Analysis
}

\section{Mingmin Huang}

Guangdong Pharmaceutical University

\section{Shaoru Chen}

Guangdong Pharmaceutical University

\section{Kening Zheng}

Guangdong Pharmaceutical University

Qu Liu

Guangdong Pharmaceutical University

\section{Kening Li}

China Pharmaceutical University

\section{Minghua Xian}

Guangdong Pharmaceutical University

Shumei Wang ( $\nabla$ gdpuwsm@126.com )

Guangdong Pharmaceutical University

\section{Research}

Keywords: Ginsenoside Rc, neuroprotective effect, oxygen-glucose deprivation reperfusion (OGD/R) injuries, TNF-a; DRP-1

Posted Date: August 3rd, 2020

DOI: https://doi.org/10.21203/rs.3.rs-51034/v1

License: (9) This work is licensed under a Creative Commons Attribution 4.0 International License. Read Full License 


\section{Abstract}

Background: Ginsenoside Rc (Rc) is one of the major active components of Panax ginseng Meyer. Studies have shown that Rc has remarkable effect in protection of nervous system. However, the potential molecular mechanism of its neuroprotective effect remains unclear. Our study aim to investigate the neuroprotective effect of Rc on neuron damage and explore the potential mechanism on its regulation of TNF- $a$ and DRP-1.

Methods: Oxygen-glucose deprivation reperfusion (OGD/R) cell neuron damage modle was induced by $\mathrm{Na}_{2} \mathrm{~S}_{2} \mathrm{O}_{4}$ and EBSS solution. After preventive administration, cell viability and cell toxicity were detected to evaluate the putative neuroprotective properties of Rc. Network pharmacology and molecular docking simulation studies were performed to predict the potential targets and pharmacological mechanism. Furthermore, the prediction was validated via western blot assay and specific antagonist.

Results: In OGD/R injured cells, Rc significantly improved cell viability (Rc middle dose vs. OGD/R model: $67.3 \pm 2.33 \%$ vs. $55.7 \pm 1.14 \%, P<0.05$ ) and obviously decreased cell toxicity (Rc middle dose vs. OGD/R model: $147 \pm 39.7 \%$ vs. $232 \pm 29.4 \%, P<0.01)$. Analysis of network pharmacology and molecular docking indicated that the key targets of Rc are TNF-a and DRP-1. Subsequently molecular biological studies showed a significant increase on expression of TNF-a and DRP-1 in model group. Conversely, administration of Rc reversed the alteration significantly and presented a dose dependence. By adding antagonist, we validated that Rc had an indirect regulation on TNF- $a$ and DRP-1.

Conclusions: Rc possess protective properties against OGD-induced neuron damage by regulating the expression of TNF- $a$ and DRP-1.

\section{Background}

Stroke is a brain injured disease with high incidence, death, and disability rates. The global burden of disease in 2016 showed that stroke is the leading cause of life years loss in China[1,2]. In clinic, ischemic stroke (IS) accounts more than $85 \%$ of stroke. For the past two decades, the mainstay of acute ischemic stroke (AIS) management has been attempted reperfusion of ischemic tissue with intravenous thrombolysis[3]. Until now, alteplase, one of the recombinant tissue plasminogen activator, is the only fibrinolytic agent with Food and Drug Administration (FDA)-approved for AIS treatment. However, there were less than $3 \%$ patients who can benefit from intravenous thrombolysis because of the strict therapeutic time window limit and fatal side effects[4]. Besides, fibrinolytic therapy may cause ischemiareperfusion (I/R) injury, which results in high morbidity and high mortality[5]. Currently, neuroprotective therapies have shown the potential to prolong the therapeutic window for reperfusion prior to endovascular interventions[6]. However, neuroprotection for stroke has shown great promise but has had little translational success. Therefore, the development of a clinically effective and safe neuroprotective drug for the treatment of ischemic stroke remains an urgent unmet need. 
Ginsenoside Rc (Rc) is a major natural product isolated from Panax ginseng Meyer, which is widely used as both a preventive and therapeutic treatment against various diseases. Rc has exhibited effects on protection of central nervous system[7,8], prevention of diabetes[9], anti-inflammatory[10], antioxidation[11] and anti-adipogenesis[12]. In addition, Rc can be absorbed into blood and brain tissue rapidly after administration[13]. Researches have shown that ginsenosides are effective in treating cerebral I/R and other nervous system diseases[14]. Resently, some ginsenosides are regarded as neuroprotective agents to attenuate IS damages[15,16]. However, although the pharmacological activities of Rc have been well studied, it remains not clear whether RC has neuroprotective effect in IS. Moreover, the specific mechanisms have not been fully elucidated. From a therapeutic perspective, it is important to understand the functional mechanisms of RC to be developed into clinically effective and safe neuroprotective drug.

Network pharmacology is a prospective strategy to explore the multi-targets regulation networks of chemicals with combining systematic methods[17]. Until now, it has been reported in lots of researches on exploring the molecular mechanisms of effective components from TCM herbs[18]. Integrative Pharmacology-based Research Platform of Traditional Chinese Medicine (TCMIP) is an intelligent data mining platform which has been extensively used to explain the functional mechanisms of TCM[19,20]. Besides, molecular docking is a computational method for predicting the placement of ligands in the binding sites of their receptors. It has been widely used in drug discovery[21]. Oxygen-glucose deprivation(OGD/R) cell modelhas been well recognized for stroke in vitro. Therefore, this study aimed to investigate the neuroprotective potential of Rc and explore the mechanism for treatment on IS via methods of network pharmacology, including target prediction of chemical, topological feature analysis, and molecular docking. Then the putative potential targets of Rc in OGD/R injury will be validated by molecular biology approach.

\section{Materials And Methods}

\section{Chemicals and reagents}

Ginsenoside Rc (purity, $\geq 98 \%$ ) was obtained from Chengdu Chroma-Biotechnology Co., Ltd. (Chengdu, China). Cell Counting Kit-8 (CCK-8) was from Dojindo Institute (Kumamoto, Japan). Lactate Dehydrogenase (LDH) Activity Assay Kit was purchased from Nanjing Jiancheng Bioengineering Institute (Nanjing, China). Bicinchoninic Acid (BCA) Kit for Protein Determination was purchased from NanJing KeyGen Biotech Co.,Ltd. (Nanjing, China). Cell lysis buffer was obtained from Promega Corporation (Madison, WI, USA).

\section{Cell culture}

PC12 cell line was obtained from Shanghai Institute of Cell Biology, Chinese Academy of Sciences (Shanghai, China). Cells were cultured in high-glucose Dulbecco's modifed Eagle's medium (DMEM; GIBCO, Waltham, MA, USA) supplemented with $10 \%$ heat inactivated fetal bovine serum (FBS; GIBCO, 
Waltham, MA, USA), $100 \mathrm{U} / \mathrm{ml}$ penicillin and $100 \mu \mathrm{g} / \mathrm{ml}$ streptomycin, and maintained at $37^{\circ} \mathrm{C}$ in a humidifed atmosphere with $5 \% \mathrm{CO}_{2}$.

\section{Cell treatment and OGD/R model}

Cells were seeded in 96-well plate at a density of $6 \times 10^{3}$ cells/well and incubated for 24 hours. Thereafter, Nimodipine (positive drug) group was treated with $5 \mu \mathrm{mol} / \mathrm{L}$ Nimodipine. Treatment groups were treated with 1000,500 , and $100 \mu \mathrm{mol} / \mathrm{L} \mathrm{Rc}$, respectively. While control group and model group were just cultured in normal medium. After culturing for 24 hours, Earle's Balanced Salts (EBSS) with $5 \mathrm{mmol} / \mathrm{L} \mathrm{Na}{ }_{2} \mathrm{~S}_{2} \mathrm{O}_{4}$ was added into groups except control group. After culturing for 80 minutes, the medium was replaced by normal DMEM with glucose and cells were cultured for 24 hours for reoxygenation under normoxic condition.

\section{Preparation of cell lysates}

Adherent cells were harvested using a cell scraper, washed twice with PBS and maintained on ice for 5 min. Following centrifugation at $14,000 \mathrm{rpm}$ for $15 \mathrm{~min}$ at $4^{\circ} \mathrm{C}$, the cell pellets were dissolved in cell lysis buffer containing $1 \mu \mathrm{L}$ PMSF and Cocktail (Sigma-Aldrich; Merck KGaA), and maintained on ice for 30 min. Following centrifugation at $10,000 \mathrm{rpm}$ for $5 \mathrm{~min}$ at $4^{\circ} \mathrm{C}$, the supernatants were collected. Protein concentration in cell lysates was determined by the BCA kit assay according to the manufacturer's instructions.

\section{Cell viability and toxicity assay.}

In order to investigate the putative protective properties of Rc in OGD on PC12 cells, cell viability was evaluated using a CCK-8 assay, and cell toxicity was determined by LDH assay. After treatment, CCK-8 assay and LDH assay were performed according to the manufacturer's instructions as described previously[22].

\section{Target prediction}

Identifcation of targets of Rc is a key step in understanding its mechanisms. In this study, the methods,includingTCMIP[19] (http://www.tcmip.cn/TCMIP/index.php/Home/Login/login.html),was used to derive molecular target information for target prediction. TCMIP is a new powerful platform for predicting targets of actual bioactive ingredients that based on similarity ensemble analysis.

\section{Functional analysis of the putative targets by STRING.}

The STRING database (https://string-db.org/), including data on interacting proteins or genes in humans, was used to determine protein-protein interactions (PPI). Interactions among the putative targets were identified using a threshold score of 0.7 . To mine the critical targets related to ischemic stroke, a targetfunction network of ischemic stroke was constructed, with the target-function relation based on the network topological analysis by Cytoscape 3.7.1. 


\section{Molecular docking of Rc.}

Molecular docking was analyzed using the SYBYL-X 2.1.1 software (Certara, L. P.). The scoring function total-Score equal to 5 was used as a threshold to evaluate the interaction between ingredients and targets. The structure of disease targets employed in the analysis of docking was obtained from the Protein Data Bank (PDB, http://www.rcsb.org). The co-crystalized ligand and water molecules were removed from the structure, while $\mathrm{H}$ atoms were added and side chains were fixed during protein preparation. The Surflex-Dock (SFXC) docking mode was used, and the procedure was conducted as previously described. Total Surflex-Dock scores represent binding affinities.

\section{Western blot analysis.}

Cellular lysates (protein content, $20 \mu \mathrm{g} / \mathrm{lane}$ ) were separated using SDS PAGE on a 10\% (w/v) gel and electrotransferred to a polyvinylidene fluoride membrane (Millipore; USA). The membranes, after blocking with $5 \%$ nonfat milk (Sigma-Aldrich; Merck KGaA) for 1 hour at room temperature, were probed with the primary antibodies at $4^{\circ} \mathrm{C}$ overnight, and subsequently incubated with horseradish peroxidase-conjugated secondary antibodies (TNF-a Rabbit Antibody, ab11564; DRP-1 Rabbit Antibody, ab184247; Abcam, UK) at room temperature for 2 hours. Protein bands were visualized by using the ECL western detection reagent. GAPDH was used as the loading control. Densities of the protein bands were determined using ImageJ2x software.

\section{Statistical analysis.}

Data are expressed as the mean \pm standard deviation. The statistical signifcance of the differences between groups was assessed using One-way analysis of variance. Graphpad Prism version 8.0.1 for Windows was used to perform the statistical analyses. $P<0.05$ was considered to indicate a statistically signifcant difference.

\section{Results}

\section{Effects of ginsenoside Rc on cell viability and toxicity in OGD/R injured cells.}

To evaluate the putative neuroprotective properties of Rc in OGD/R injured cells, the cell viability was detected by CCK-8 assay and the cytotoxicity was determined by LDH leakage. As shown in Fig.1A, compared with control group, the cell viability in model group $\varangle 55.7 \pm 1.14 \% \bigotimes$ indicated impairment $\left({ }^{\# \#} \mathrm{P}<0\right.$. 01). Compared with model group, the cell viability in various concentrations of Rc groups $₫$ high-dose group: $67.1 \pm 2.83 \%$; middle-dose group: $67.3 \pm 2.33 \%$; low-dose group: $66.5 \pm 4.76 \% \bigotimes$ were significantly improved ( $\left.{ }^{*} P<0.05\right)$. Furthermore, the LDH leakage in OGD/R injured cells was determined as shown in Fig.1B. The LDH leakage was obviously enhanced in model group (232 $\pm 29.4 \%)$, as compared with control group $\left(179 \pm 51.2 \%\right.$; $\left.{ }^{\# \#} \mathrm{P}<0.01\right)$. Rc treatment could inhibit the $\mathrm{LDH}$ release thigh-dose group: $180 \pm 37.4 \%$; middle-dose group: $147 \pm 39.7 \%$; low-dose group: $166 \pm 34.1 \% \square$ compared to model group 
$\left({ }^{*} \mathrm{P}<0.01\right)$. The result showed that Rc improved cell viability and reduced the toxicity of LDH leakage in OGD/R injured cells.

\section{Target prediction.}

Eighteen potential targets of Rc were predited by TCMIP database. Then protein-protein interactions of these targets were determined by The STRING database as shown in Fig.2B. As revealed in Fig.2C, the enrichment of GO was performed by STRING. The enrichment of functional pathway was found out that the top 10 of pathway, such as TNF signaling pathway, is illustrated in Fig.2D. The network topological analysis by Cytoscape demonstrated that targets with the top five scores of degree were TNF. The key pathway of Rc maybe the TNF signal pathway.

The targets were analysed in Cytoscape and ranked by degree as shown in Table 1. The top 4 were TNF/CASPA3/NFKB1/IL6.

Table 1. The results of network topological analysis by Cytoscape

\begin{tabular}{|llll|}
\hline Name & Degree & BetweennessCentrality & ClosenessCentrality \\
\hline TNF & 10 & 0.18861111 & 0.69565217 \\
\hline CASP3 & 10 & 0.18680556 & 0.72727273 \\
\hline NFKB1 & 10 & 0.13583333 & 0.72727273 \\
\hline IL6 & 9 & 0.10513889 & 0.69565217 \\
\hline IL1B & 8 & 0.06305556 & 0.61538462 \\
\hline NR3C1 & 8 & 0.07291667 & 0.61538462 \\
\hline NFKB2 & 7 & 0.00333333 & 0.59259259 \\
\hline RIPK3 & 6 & 0.02902778 & 0.57142857 \\
\hline ROCK1 & 5 & 0.15833333 & 0.55172414 \\
\hline VDR & 4 & 0.04444444 & 0.51612903 \\
\hline
\end{tabular}

\section{Key targets of the Rc in TNF signal pathway}

To identify the key targets of Rc, the targets in TNF pathway related to ischemic stroke were imported into SYBYL to find out key target. The results of molecular docking are shown in Fig.3A. As depicted in Fig.3A, $\mathrm{RC}$ is bound to residues outside the active pocket of TNF (key residues including ARG201/ASN200/ASP254/ASN182/ARG143/ASP294/ARG295)by hydrogen bonds. The overall spatial 
structure indicates that the TNF/Rc complexes are stable, indicating the interactions of Rc with their targets may have an active functional role.

Referring to literature on ischemic stroke, TNF signal pathway (As shown in Fig.3B), including TNF, DRP1, play important role in necroptosis.

\section{Effect of ginsnoside Rc on the expression of TNF-a/DRP-1 in OGD/R injured cells}

To explore the effect on TNF and DRP-1 by RC, as shown in Fig.4, the result revealed that Rc decreased the protein level of TNF- $a$ and DRP-1 in OGD/R injured cells. There was a significant increase in expression of TNF- $a$ and DRP-1 of model group compared with control group ( $* * P<0.01)$. Conversely, administration of different concentrations of Rc resulted in an extremely significant decrease in protein level of TNF- $\alpha$ and DRP- 1 with a certain dose dependence compared with model group $(* * P<0.01)$.

\section{Effects on cell survival rate induced by ginsnoside Rc and EvP4593/Mdivi-1}

To further determine the correlation between Rc and TNF-a/DRP-1, cells were treated by EvP4593 (antagonist of TNF-a) and Mdivi-1 (antagonist of DRP-1), respectively. Cell survival rate was detected by CCK-8 assay. The result was shown in Fig.4D. Compared with Rc group (65.87 $\pm 9.67 \%)$, the survival rate of Rc+EvP4593 (57.42 $\pm 5.22 \%)$ was tended to decrease. Compared with model group, the survival rate of EvP4593 group showed no significant difference. The result indicated that Rc may had an indirect effect on TNF-a. Besides, the survival rate of Rc+Mdivi-1 showed no significant difference with Rc group. There was also no significant difference between model group and Mdivi-1 group, which indicated that Rc may also had an indirect effect on DRP-1.

\section{Discussion}

Ischemic stroke is a complex multifactorial disease caused by infarction and result in the loss of neurologic function. For decades, the most effective way to treat cerebral infarction is fibrinolytic therapy[23]. However, there isn't any effective treatment for stroke caused by neuronal damage and death. In this study, we investigated the putative neuroprotective properties of Rc and the molecular machanism, which showed a positive significance for development of neuroprotective drugs for the treatment of cerebral I/R injury to reduce safety concerns caused by antithrombotic drugs in IS.

In our study, we found that pretreatment of Rc enhanced the cell viability and reduced the cell toxicity on OGD/R injured cells (Fig.1), which provided evidence for Rc as one of the active components of P. ginseng.Then, network pharmacology and molecular docking were applied to predict the related targets and corresponding mechanisms of Rc in treatment of IS. A protein-protein interaction network based on Rc was constructed. Bynetwork topological analysis, we revealed and highlighted that Rc were involved in TNF signal pathway (Fig.2). Analysis of molecular docking and literature on IS indicated that TNF signal pathway (Fig.3), including TNF, DRP-1, play important role in IS. The correlation between Rc and predicted targets was explored via western blots. Results showed that Rc reversed the expression of TNF-a and 
DRP-1 on OGD/R injured cells when compared with model group (Fig.4). To further explore whether Rc possess neuroprotective effect by directly targeting TNF- $a$ and DRP-1, the antagonists were added as interfering agent respectively. The result showed that Rc had an indirect effect on TNF- $a$ and DRP-1 (Fig.4). In terms of experimental method, network pharmacology and molecular docking are not enough to judge the targets of Rc in IS. So cell experiments were combined in this study for illustration. This work may has practical significance for rapidly discovery on related targets and corresponding mechanisms of monomer drugs.

The tumor necrosis factor (TNF) superfamily of cytokines activate signaling pathways plays an important role in cell survival, death, and differentiation. A network pharmacology research had indicated that the treatment of compounds on stroke may related to TNF signaling pathway and TNF-a may be one of the molecular markers for stroke[24]. TNF-a is a pleiotropic inflammatory cytokine cytokine with various biological functions, which is mainly produced by macrophages and monocyte. TNF-a participates in several immunity disease and inflammatory disease[25], and it is a crucial determinant of inflammatory reaction in stroke[26]. Studies have shown that the expression of TNF-a after cerebral I/R has neurotoxic effect on nervous system[27]. Moreover, it was suggested that TNF-a may play a neurorotective role in stroke by downregulating apoptosis[28]. In addition, we found that stroke may be related to NFKB1 and CASP3 (table 1), which is consistant with the existing research finding[29]. It is well known that TNF-a plays a critical role on the activation of NFKB and caspase-3[30,31], which also provided evidence to illustrate TNF-a as a key target.

Dynamin-related protein-1 (DRP-1) is the dynamin of mitochondrial fission[32]. The imbalance of mitochondrial fusion/division has great influence on I/R injuries[33]. Previous studies had shown that TNF- $a$ and DRP-1 played a regulatory role in amelioration of cerebral ischemic injury and neuroinflammation[34]. Moreover, TNF-a is the predominant inducer of DRP1 S616 phosphorylation during sepsis[35]. DRP-1 had neuroprotective effect in OGD-induced hippocampal neurons[36]. The overexpression of DRP-1 in nerve cells will eventually lead to cell apoptosis and mitochondrial lysis through activation of caspase system and damage of important proteins and organelles in mitochondria[37]. Our study document and validation suggested that Rc ameliorates neuron damage on oxygen-glucose deprivation associated with regulating TNF-a and DRP-1. The neuroprotective effects of Rc may be related to inflammatory response and necroptosis during nerve cell injury.

\section{Conclusions}

This study identified the neuroprotective activity of ginsenoside Rc mediated by its regulation on TNF-a and DRP-1. Rc may be involved in the pathological process of cell necroptosis and inflammatory. This work provide a step forward in the understanding of the neuroprotective effect and underlying mechanism of Rc on OGD induced neuron damage. However, the current study is performed based on in vitro experiments and the conclusions remain to beconfrmed by in vivo experiments.

\section{List Of Abbreviations}




\begin{tabular}{|ll|}
\hline Rc & Ginsenoside Rc \\
\hline IS & ischemic stroke \\
\hline I/R & ischemia-reperfusion \\
\hline TCMIP & Integrative Pharmacology-based Research Platform of Traditional Chinese Medicine \\
\hline OGD/R & oxygen-glucose deprivation reperfusion \\
\hline TNF-a & Tumor necrosis factor-a \\
\hline Drp-1 & Dynamin-related protein-1 \\
\hline
\end{tabular}

\section{Declarations}

\section{Ethics approval and consent to participate}

Not applicable.

\section{Consent for publication}

This manuscript is approved by all authors for publication.

\section{Availability of data and materials}

The datasets generated for this study are available on request to the corresponding author.

\section{Competing interests}

The authors declare that they have no competing interests.

\section{Funding}

This research is supported by Projects of The National Natural Science Foundation of China (grant numbers 81773884, 81473431,81274060)and the National Science and Technology Major Project (grant numbers 2017ZX09301077).

\section{Authors' contributions}

Mingmin Huang and Shaoru Chen contributed equally to this manuscript.

$\mathrm{MX}$ and SW conceived and designed the idea; $\mathrm{KL}$ performed the experiments; $\mathrm{MH}$ and SC analyzed the data; KZ and QL performed network pharmacology analysis; $\mathrm{MX}$ and $\mathrm{MH}$ wrote the paper. All authors read and approved the final manuscript.

\section{Corresponding authors}


Correspondence to Minghua Xian or Shumei Wang.

\section{Acknowledgements}

Technology Funds were obtained from the Key Unit of Chinese Medicine Digitalization Quality Evaluation of State Administration of Traditional Chinese Medicine. We thank Haibin Luo from Sun Yat-sen University for technical assistance in SYBYL.

\section{Reference}

1. GBD 2016 Causes of Death Collaborators. No Title. Lancet (London, England) [Internet]. Lancet; 2017 [cited 2020 Jul 13];390:1151-210. Available from: http://www.ncbi.nlm.nih.gov/pubmed/28919116

2. Zhou M, Wang H, Zhu J, Chen W, Wang L, Liu S, et al. Cause-specific mortality for 240 causes in China during 1990-2013: a systematic subnational analysis for the Global Burden of Disease Study 2013. Lancet (London, England) [Internet]. Lancet; 2016 [cited 2020 Jul 15];387:251-72. Available from: http://www.ncbi.nlm.nih.gov/pubmed/26510778

3. Phipps MS, Cronin CA. Management of acute ischemic stroke. BMJ [Internet]. BMJ; 2020 [cited 2020 Jul 15];368:16983. Available from: http://www.ncbi.nlm.nih.gov/pubmed/32054610

4. Emberson J, Lees KR, Lyden P, Blackwell L, Albers G, Bluhmki E, et al. Effect of treatment delay, age, and stroke severity on the effects of intravenous thrombolysis with alteplase for acute ischaemic stroke: a meta-analysis of individual patient data from randomised trials. Lancet (London, England) [Internet]. Lancet; 2014 [cited 2020 Jul 15];384:1929-35. Available from:

http://www.ncbi.nlm.nih.gov/pubmed/25106063

5. Saito M, Chen-Yoshikawa TF, Suetsugu K, Okabe R, Takahagi A, Masuda S, et al. Pirfenidone alleviates lung ischemia-reperfusion injury in a rat model. J Thorac Cardiovasc Surg [Internet]. J Thorac Cardiovasc Surg; 2019 [cited 2020 Jul 13];158:289-96. Available from:

http://www.ncbi.nlm.nih.gov/pubmed/30385019

6. SI S, JC B, MA Y, N S, M F. Reconsidering Neuroprotection in the Reperfusion Era. Stroke [Internet]. Stroke; 2017 [cited 2020 Jul 15];48. Available from: http://www.ncbi.nlm.nih.gov/pubmed/29146878

7. Liu J, Chen F, Yin J, Bu F, Zheng B, Yang M, et al. The effects of ginsenosides to amyloid fibril formation by RCMк-casein. Int J Biol Macromol [Internet]. Int J Biol Macromol; 2015 [cited 2020 Jul 15];79:49-55. Available from: http://www.ncbi.nlm.nih.gov/pubmed/25934110

8. RJ C, A R, HJ J, MY A, BS M, CH P, et al. BACE1 molecular docking and anti-Alzheimer's disease activities of ginsenosides. J Ethnopharmacol [Internet]. J Ethnopharmacol; 2016 [cited 2020 Jul 15];190. Available from: http://www.ncbi.nlm.nih.gov/pubmed/27275774 
9. MS L, JT H, SH K, S Y, MS K, HJ Y, et al. Ginsenoside Rc, an active component of Panax ginseng, stimulates glucose uptake in $\mathrm{C} 2 \mathrm{C} 12$ myotubes through an AMPK-dependent mechanism. $\mathrm{J}$ Ethnopharmacol [Internet]. J Ethnopharmacol; 2010 [cited 2020 Jul 18];127. Available from: http://www.ncbi.nlm.nih.gov/pubmed/19961916

10. Yu T, Yang Y, Kwak Y-S, Song GG, Kim M-Y, Rhee MH, et al. Ginsenoside Rc from Panax ginseng exerts anti-inflammatory activity by targeting TANK-binding kinase 1 /interferon regulatory factor-3 and p38/ATF-2. J Ginseng Res [Internet]. J Ginseng Res; 2017 [cited 2020 Jul 15];41:127-33. Available from: http://www.ncbi.nlm.nih.gov/pubmed/28413316

11. Kim DH, Park CH, Park D, Choi YJ, Park MH, Chung KW, et al. Ginsenoside Rc modulates Akt/FoxO1 pathways and suppresses oxidative stress. Arch Pharm Res [Internet]. Arch Pharm Res; 2014 [cited 2020 Jul 15];37:813-20. Available from: http://www.ncbi.nlm.nih.gov/pubmed/23918648

12. Zhang Y, Lin L, Liu GY, Liu JX, Li T. Pharmacokinetics and brain distribution of ginsenosides after administration of sailuotong. Chin J Chin Mater Med 2014, 39(2):316-321. Available from: https://www.cnki.net/

13. Guo Q, Li P, Wang Z, Cheng Y, Wu H, Yang B, et al. Brain distribution pharmacokinetics and integrated pharmacokinetics of Panax Notoginsenoside R1, Ginsenosides Rg1, Rb1, Re and Rd in rats after intranasal administration of Panax Notoginseng Saponins assessed by UPLC/MS/MS. J Chromatogr B Analyt Technol Biomed Life Sci [Internet]. 2014 [cited 2020 Jul 15];969:264-71. Available from: https://linkinghub.elsevier.com/retrieve/pii/S1570023214005510

14. Zheng M, Xin Y, Li Y, Xu F, Xi X, Guo H, et al. Ginsenosides: A Potential Neuroprotective Agent. Biomed Res Int [Internet]. Biomed Res Int; 2018 [cited 2020 Jul 13];2018:8174345. Available from: http://www.ncbi.nlm.nih.gov/pubmed/29854792

15. Ahmed T, Raza SH, Maryam A, Setzer WN, Braidy N, Nabavi SF, et al. Ginsenoside Rb1 as a neuroprotective agent: A review. Brain Res Bull [Internet]. Brain Res Bull; 2016 [cited 2020 Jul 13];125:3043. Available from: http://www.ncbi.nlm.nih.gov/pubmed/27060612

16. Nabavi SF, Sureda A, Habtemariam S, Nabavi SM. Ginsenoside Rd and ischemic stroke; a short review of literatures. J Ginseng Res [Internet]. J Ginseng Res; 2015 [cited 2020 Jul 13];39:299-303. Available from: http://www.ncbi.nlm.nih.gov/pubmed/26869821

17. M S, C G, M L, X L, B Y. Therapeutic targets of vitamin $C$ on liver injury and associated biological mechanisms: A study of network pharmacology. Int Immunopharmacol [Internet]. Int Immunopharmacol; 2019 [cited 2020 Jul 15];66. Available from: http://www.ncbi.nlm.nih.gov/pubmed/30530052

18. B Z, W Z, Y L, S H, R G, Z S, et al. Network pharmacology-based identification of protective mechanism of Panax Notoginseng Saponins on aspirin induced gastrointestinal injury. Biomed Pharmacother 
[Internet]. Biomed Pharmacother; 2018 [cited 2020 Jul 15];105. Available from: http://www.ncbi.nlm.nih.gov/pubmed/29857294

19. Xu HY, Zhang YQ, Liu ZM, Chen T, Lv CY, Tang SH, et al. ETCM: An encyclopaedia of traditional Chinese medicine. Nucleic Acids Res [Internet]. Nucleic Acids Res; 2019 [cited 2020 Jul 15];47:D976-82. Available from: http://www.ncbi.nlm.nih.gov/pubmed/30365030

20. Su J, Zhou R-R, Guo F-F, Zhou Q-W, Xu H-Y. [Molecular mechanism of Shuguan Granules in treating angina based on integrative pharmacology]. Zhongguo Zhong Yao Za Zhi [Internet]. Zhongguo Zhong Yao Za Zhi; 2019 [cited 2020 Jul 13];44:1425-35. Available from:

http://www.ncbi.nlm.nih.gov/pubmed/31090301

21. Pinzi L, Rastelli G. Molecular Docking: Shifting Paradigms in Drug Discovery. Int J Mol Sci [Internet]. Int J Mol Sci; 2019 [cited 2020 Jul 13];20. Available from:

http://www.ncbi.nlm.nih.gov/pubmed/31487867

22. Duan Y, Cheng S, Jia L, Zhang Z, Chen L. PDRPS7 protects cardiac cells from hypoxia/reoxygenation injury through inactivation of JNKs. FEBS Open Bio [Internet]. FEBS Open Bio; 2020 [cited 2020 Jul 13];10:593-606. Available from: http://www.ncbi.nlm.nih.gov/pubmed/32108998

23. Armahizer M, Blackman A, Plazak M, Brophy GM. Early Acute Ischemic Stroke Management for Pharmacists. Hosp Pharm [Internet]. Hosp Pharm; 2020 [cited 2020 Jul 13];55:12-25. Available from: http://www.ncbi.nlm.nih.gov/pubmed/31983762

24. Wu ZM, Guo LN, Wang DD. Network pharmacological study on Astragalus Rhizoma-Chuanxiong drug pair in treatment of stroke. Drug Evaluation Research 2019 (9):1749-1756. Available from:

https://www.cnki.net/

25. Fatani SH, ALrefai AA, Al-Amodi HS, Kamel HF, Al-Khatieb K, Bader H. Assessment of tumor necrosis factor alpha polymorphism TNF-a-238 (rs 361525) as a risk factor for development of acute kidney injury in critically ill patients. Mol Biol Rep [Internet]. Mol Biol Rep; 2018 [cited 2020 Jul 13];45:839-47.

Available from: http://www.ncbi.nlm.nih.gov/pubmed/29978383

26. R J, G Y, G L. Inflammatory Mechanisms in Ischemic Stroke: Role of Inflammatory Cells. J Leukoc Biol [Internet]. J Leukoc Biol; 2010 [cited 2020 Jul 13];87. Available from:

http://www.ncbi.nlm.nih.gov/pubmed/20130219

27. A V, S M, MM A, A R-P. Pentoxifylline Attenuates TNF-a Protein Levels and Brain Edema Following Temporary Focal Cerebral Ischemia in Rats. Brain Res [Internet]. Brain Res; 2011 [cited 2020 Jul 13];1377. Available from: http://www.ncbi.nlm.nih.gov/pubmed/21219888

28. Barone FC, Arvin B, White RF, et al. Tumor necrosis factor-alpha. A mediator of focal ischemic brain injury. Stroke. 1997;28(6):1233-1244. doi:10.1161/01.str.28.6.1233. Available from: 
https://pubmed.ncbi.nIm.nih.gov/9183357/

29. Wang $\mathrm{XH}$, Wang D. Effects of nimotop on NF-KB and caspase-3 expression in rat brain tissue after cerebral ischemic reperfusion. Chin J Biochem Pharm 2015. Available from: https://www.cnki.net/

30. LIU M, LIU XW, LIU XM, LIU SB, WANG Y. The correlation of TNF-a and Caspase-3 in nerve cell apoptosis after spinal cord injury of rats. Clinical $\mathrm{J}$ of Medical Officers 2017. Available from: https://www.cnki.net/

31. Morris G, Walker AJ, Berk M, Maes M, Puri BK. Cell Death Pathways: a Novel Therapeutic Approach for Neuroscientists. Mol Neurobiol [Internet]. Mol Neurobiol; 2018 [cited 2020 Jul 13];55:5767-86. Available from: http://www.ncbi.nlm.nih.gov/pubmed/29052145

32. RJ Y, AM van der B. Mitochondrial Fission, Fusion, and Stress. Science [Internet]. Science; 2012 [cited 2020 Jul 13];337. Available from: http://www.ncbi.nlm.nih.gov/pubmed/22936770

33. C H, Y H, L L. Drp1-Dependent Mitochondrial Fission Plays Critical Roles in Physiological and Pathological Progresses in Mammals. Int J Mol Sci [Internet]. Int J Mol Sci; 2017 [cited 2020 Jul 13];18. Available from: http://www.ncbi.nlm.nih.gov/pubmed/28098754

34. K Z, J C, J W, Q W, C J, YXZ X, et al. Atractylenolide III Ameliorates Cerebral Ischemic Injury and Neuroinflammation Associated With Inhibiting JAK2/STAT3/Drp1-dependent Mitochondrial Fission in Microglia. Phytomedicine [Internet]. Phytomedicine; 2019 [cited 2020 Jul 13];59. Available from: http://www.ncbi.nlm.nih.gov/pubmed/30981186

35. YL S, YZ S, GG C, LL W, MZ Z, HF J, et al. TNF-a Induces Drp1-mediated Mitochondrial Fragmentation During Inflammatory Cardiomyocyte Injury. Int J Mol Med [Internet]. Int J Mol Med; 2018 [cited 2020 Jul 13];41. Available from: http://www.ncbi.nlm.nih.gov/pubmed/29336470

36. Maneechote C, Palee S, Chattipakorn SC, Chattipakorn N. Roles of mitochondrial dynamics modulators in cardiac ischaemia/reperfusion injury. J Cell Mol Med [Internet]. J Cell Mol Med; 2017 [cited 2020 Jul 13];21:2643-53. Available from: http://www.ncbi.nlm.nih.gov/pubmed/28941171

37. Chasman DI, Schürks M, Anttila V, de Vries B, Schminke U, Launer LJ, et al. Genome-wide association study reveals three susceptibility loci for common migraine in the general population. Nat Genet [Internet]. Nat Genet; 2011 [cited 2020 Jul 13];43:695-8. Available from:

http://www.ncbi.nlm.nih.gov/pubmed/21666692

\section{Figures}


A

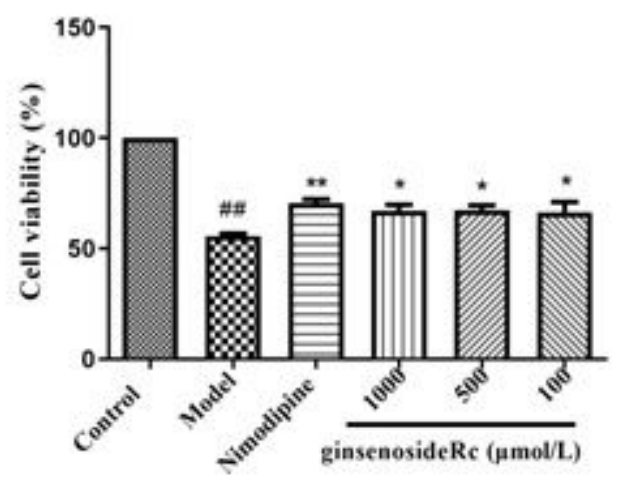

B

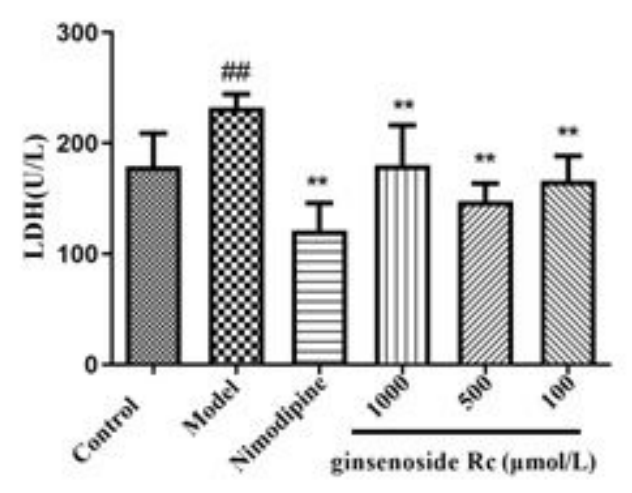

Figure 1.

\section{Figure 1}

Effects of ginsenoside Rc in OGD/R injured cells $(\bar{x} \pm s, n=6)$. (A) RC improved cell viability in OGD/R injured cells. (B) RC decreased LDH release in $O G D / R$ injured cells. \#\#P<0.01 vs control group. ${ }^{* * P}<0.01$, ${ }^{*} \mathrm{P}<0.05$ vs model group.
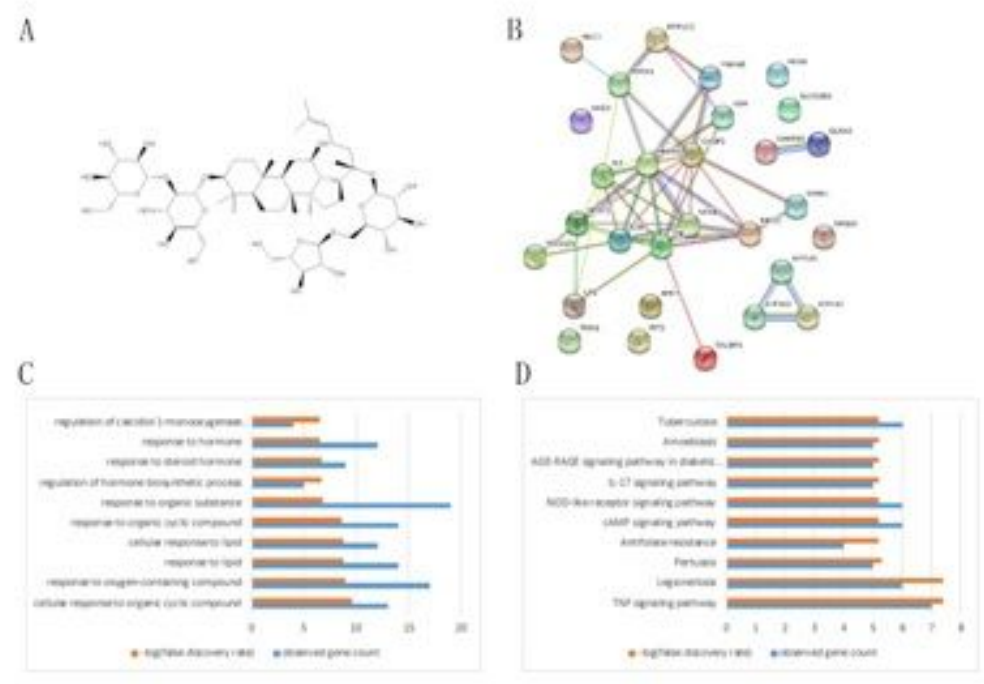

Figure. 2

Figure 2

Target prediction of RC. (A)The structure of RC. (B)Protein-protein interactions of these targets related to RC. (C) The enrichment of GO related to RC. (D) The enrichment of functional pathway related to $\mathrm{RC}$ (orange pillars represent the false discovery rate, blue pillars represent the count of targets). 
A

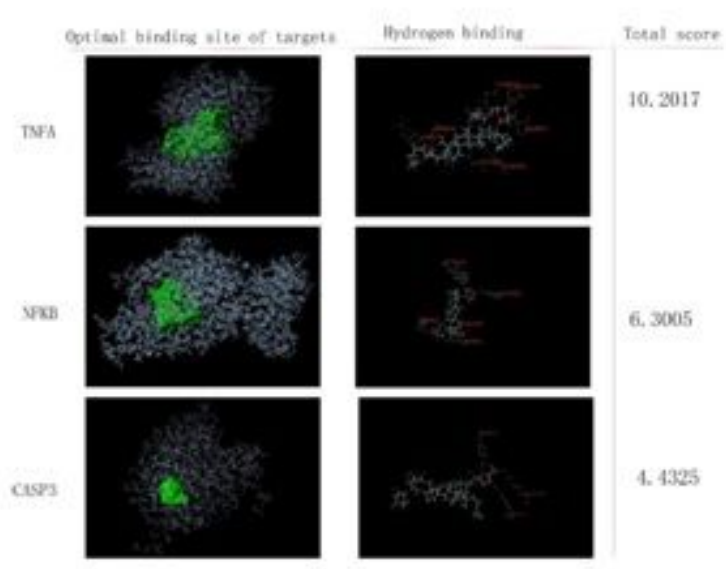

B

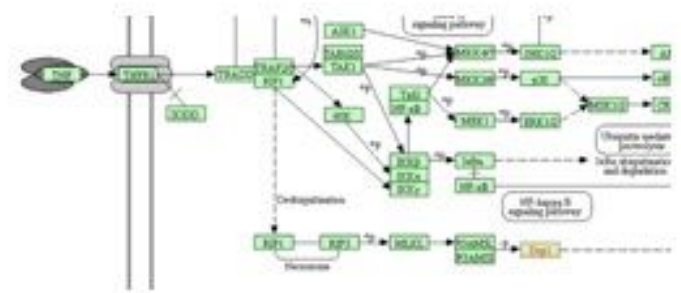

Figure.3

\section{Figure 3}

Key targets of the Rc in TNF signal pathway. (A) Molecular docking of RC with TNFA, NFKB, and CAP3.

(B) TNF signal pathway.

A

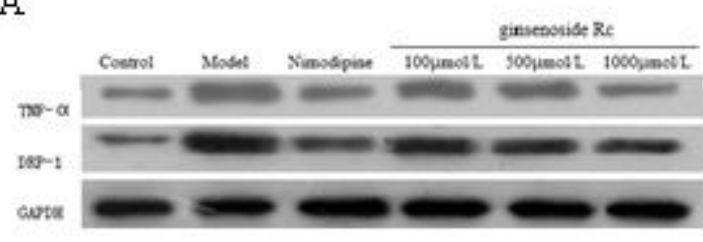

$\mathrm{C}$

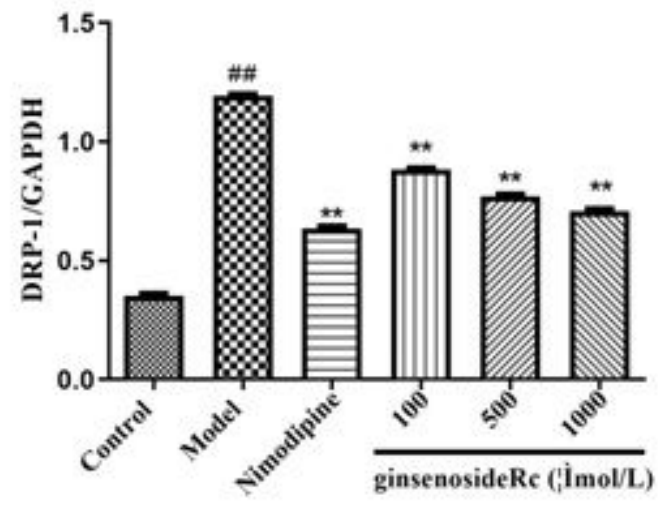

B

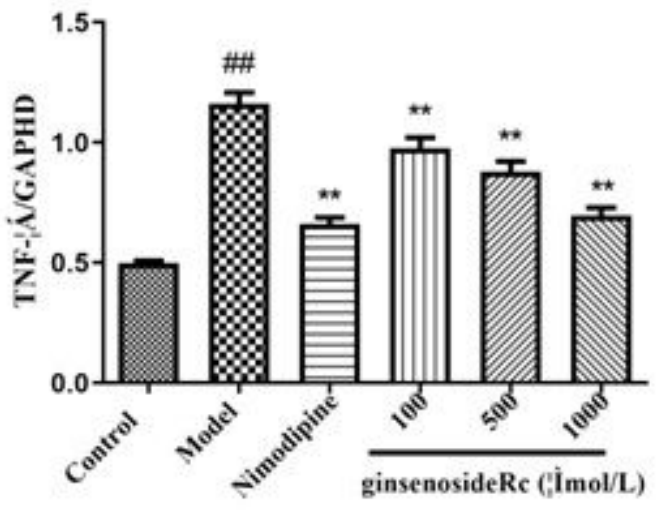

Figure 4.

Figure 4 
Protective effects of ginsenoside Rc on the protein level of TNF- $a$ /DRP-1 in OGD/R injury cells $(\overline{\mathrm{x}} \pm \mathrm{s}, \mathrm{n}=3)$. $\# \# \mathrm{P}<0.01$ vs control group. ${ }^{*} \mathrm{P}<0.01,{ }^{*} \mathrm{P}<0.05$ vs model group.

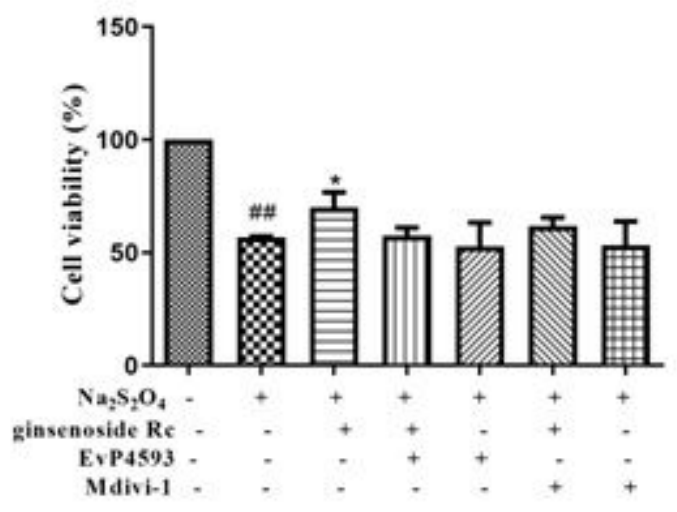

Figure 5.

\section{Figure 5}

Effects on cell survival rate induced by ginsnoside Rc and EvP4593/Mdivi-1 in OGD/R injured cells $(\overline{\mathrm{X}} \pm \mathrm{s}$, $n=6)$. \#\#P<0. 01 vs control group. ${ }^{*} P<0.01, * P<0.05$ vs model group. 